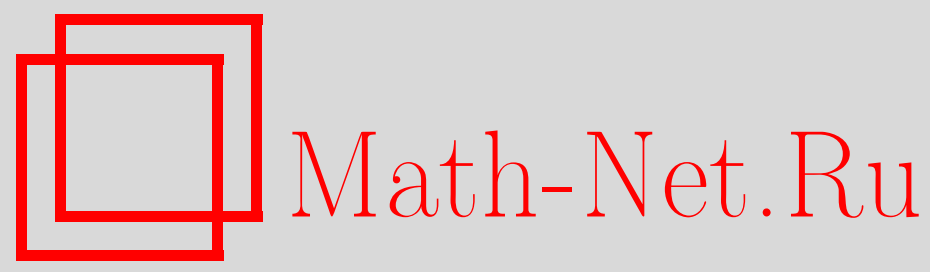

Л. С. Пулькина, Смешанная задача с интегральным условием для гиперболического уравнения, Матем. заметки, 2003, том 74, выпуск 3, 435-445

DOI: https://doi.org/10.4213/mzm277

Использование Общероссийского математического портала Math-Net.Ru подразумевает, что вы прочитали и согласны с пользовательским соглашением http://www.mathnet.ru/rus/agreement

Параметры загрузки:

IP: 54.162 .27 .143

26 апреля 2023 г., $17: 41: 44$

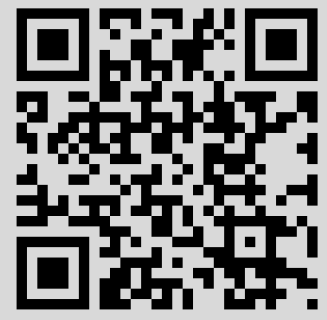


том 74 выпУСК 3 сенТяБРь 2003

УДК 517.95

\section{СМЕШАННАЯ ЗАДАЧА С ИНТЕГРАЛЬНЫМ УСЛОВИЕМ ДЛЯ ГИПЕРБОЛИЧЕСКОГО УРАВНЕНИЯ}

\section{Л. С. Пулькина}

В этой статье рассмотрена смешанная задача для гиперболического уравнения $\mathrm{c}$ граничньм условием Неймана и нелокальным интегральным условием. Обосновано утверждение о существовании единственного обобшенного решения поставленной задачи. Доказательство единственности базируется на полученной в работе априорной оценке во введенном функциональном пространстве, а существование обобщенного решения доказано методом Галеркина.

Библиография: 8 названий.

1. Постановка задачи. Рассмотрим для уравнения

$$
\mathscr{L} u \equiv u_{t t}-\left(a(x, t) u_{x}\right)_{x}+c(x, t) u=f(x, t)
$$

в области

$$
\mathscr{D}=\{(x, t): 0<x<l, 0<t<T\}
$$

задачу с начальными данными Коши

$$
u(x, 0)=\varphi(x), \quad u_{t}(x, 0)=\psi(x),
$$

граничным условием Неймана

$$
u_{x}(0, t)=0
$$

и нелокальным условием

$$
\int_{0}^{l} u(x, t) d x=0
$$

где функции $\varphi(x), \psi(x)$ заданы и удовлетворяют условиям согласования:

$$
\int_{0}^{l} \varphi(x) d x=0, \quad \int_{0}^{l} \psi(x) d x=0
$$

$a(x, t)>0$ в области $\mathscr{D}$.

Заметим, что однородность условий (3), (4) не ограничивает общности. Действительно, если $u_{x}(0, t)=\mu(t), \int_{0}^{l} u(x, t) d x=\lambda(t)$, то введя новую неизвестную функцию $\tilde{u}=u-W$, где

$$
W(x, t)=\left(x-\frac{l}{2}\right) \mu(t)+\frac{1}{l} \lambda(t),
$$


придем к задаче для уравнения $\mathscr{L} u=F(x, t)$, где $F=f-\mathscr{L} W$, с начальньми данньми

$$
\Phi(x)=\varphi(x)-\left(x-\frac{l}{2}\right) \mu(0)+\frac{1}{l} \lambda(0), \quad \Psi(x)=\psi(x)-\left(x-\frac{l}{2}\right) \mu^{\prime}(0)+\frac{1}{l} \lambda^{\prime}(0)
$$

и однородными условиями (3), (4).

Интегральные условия вида (4) встречаются при исследовании физических явлений в случае, когда граница области протекания процесса недоступна для непосредственных измерений. Примером могут служить задачи, возникающие при исследовании диффузии частиц в турбулентной плазме [1], процессов распространения тепла [2], [3], процесса влагопереноса в капиллярно-пористых средах [4].

Смешанные задачи с интегральньпи условиями для гиперболических уравнений были ранее рассмотрены в работах [5]-[7]. В [5] изучена смешанная задача для однородного уравнения колебаний струны с классическими начальными данньми и нелокальными условиями, содержащими как интегралы от искомого решения, так и его граничные значения. В [7] получено представление решения смешанной задачи для уравнения колебаний струны с граничным условием Дирихле и интегральным условием (4). В [6] рассмотрена смешанная задача для уравнения (1) с условиями (2)-(4), но для случая $c(x, t) \equiv 0$. Заметим, что в этом случае, а также если $c_{x}(x, t) \equiv 0$, задачу $(1)-(4)$ можно свести к смешанной задаче с локальными граничными условиями. Действительно, интегрируя (1) по $x$ от 0 до $l$, считая $u(x, t)$ решением поставленной задачи, получим

$$
\frac{\partial^{2}}{\partial t^{2}} \int_{0}^{l} u d x-a(l, t) u_{x}(l, t)+a(0, t) u_{x}(0, t)=\int_{0}^{l} f(x, t) d x
$$

и в силу условий $(3),(4)$

$$
a(l, t) u_{x}(l, t)=-\int_{0}^{l} f(x, t) d x .
$$

В предлагаемой работе доказано существование единственного обобщенного решения задачи $(1)-(4)$ для $c(x, t) \not \equiv 0, c_{x}(x, t) \not \equiv 0$. В этом случае интегральное условие нельзя заменить граничным.

Обозначим

$$
\begin{aligned}
& \widetilde{C}_{0}^{2}(\mathscr{D})=\left\{u(x, t): u \in C^{2}(\overline{\mathscr{D}}), \quad u_{x}(0, t)=0, \quad \int_{0}^{l} u(x, t) d x=0\right\} \\
& \widetilde{C}_{T}^{2}(\mathscr{D})=\left\{v(x, t): v \in C^{2}(\overline{\mathscr{D}}), \quad v(x, T)=0, \quad \int_{0}^{l} v(x, t) d x=0\right\} .
\end{aligned}
$$

Замыкание этих пространств по норме

$$
\|u\|_{\widetilde{\mathscr{H}}(\mathscr{D})}^{2}=\int_{0}^{T} \int_{0}^{l}\left[u^{2}+\left(\int_{x}^{l} u(\xi, t) d \xi\right)^{2}+\left(\int_{x}^{l} u_{t}(\xi, t) d \xi\right)^{2}\right] d x d t
$$

обозначим соответственно $\widetilde{\mathscr{H}}_{0}(\mathscr{D}), \widetilde{\mathscr{H}}_{T}(\mathscr{D})$.

Введем понятие обобщенного решения. Пусть $u(x, t)$ - классическое решение задачи (1)-(4). 
Рассмотрим оператор

$$
\ell v=\int_{x}^{l}(\xi-x) v(\xi, t) d \xi, \quad v(x, t) \in \widetilde{C}_{T}^{2}(\mathscr{D}) .
$$

Умножим обе части (1) на $\ell v$ и проинтегрируем по области $\mathscr{D}$. Полученное равенство преобразуем, интегрируя слева по частям:

$$
\begin{gathered}
\int_{0}^{T} \int_{0}^{l} u_{t t} \int_{x}^{l}(\xi-x) v d \xi d x d t=\left.\int_{0}^{T} \int_{x}^{l}(\xi-x) v d \xi \int_{x}^{l} u_{t t} d \xi\right|_{0} ^{l} d t \\
-\int_{0}^{T} \int_{0}^{l} \int_{x}^{l} u_{t t} d \xi \int_{x}^{l} v d \xi d x d t=\left.\int_{0}^{T} \int_{x}^{l}(\xi-x) v d \xi \int_{x}^{l} u_{t t} d \xi\right|_{0} ^{l} d t \\
-\left.\int_{0}^{l} \int_{x}^{l} u_{t} d \xi \int_{x}^{l} v d \xi\right|_{0} ^{T} d x+\int_{0}^{l} \int_{x}^{l} u_{t} d \xi \int_{x}^{l} v_{t} d \xi d x d t \\
\int_{0}^{T} \int_{0}^{l}\left(a u_{x}\right)_{x} \int_{x}^{l}(\xi-x) v d \xi d x d t=\left.\int_{0}^{T} a u_{x} \int_{x}^{l}(\xi-x) v d \xi\right|_{0} ^{l} d t \\
+\int_{0}^{T} \int_{0}^{l} a u_{x} \int_{x}^{l} v d \xi d x d t \\
=\left.\int_{0}^{T} a u_{x} \int_{x}^{l}(\xi-x) v d \xi\right|_{0} ^{l} d t+\left.\int_{0}^{T} a u \int_{x}^{l} v d \xi\right|_{0} ^{l} d t \\
-\int_{0}^{T} \int_{0}^{l} a_{x} u \int_{x}^{l} v d \xi d x d t+\int_{0}^{T} \int_{0}^{l} a u v d x d t \\
\int_{0}^{T} \int_{0}^{l} c u \int_{x}^{l}(\xi-x) v d \xi d x d t=-\left.\int_{0}^{T} c \int_{x}^{l} u d \xi \int_{x}^{l}(\xi-x) v d \xi\right|_{0} ^{l} d t \\
+\int_{0}^{T} \int_{0}^{l} c_{x}\left(\int_{x}^{l} u d \xi\right) \ell v d x d t-\int_{0}^{l} \int_{0}^{l} u \int_{x}^{l} u d \xi \int_{x}^{l} v d \xi d x d t
\end{gathered}
$$

Учитьвая, что $\int_{0}^{l} u d x=0, u_{x}(0, t)=0, u_{t}(x, 0)=\psi(x), v(x, T)=0$, получим

$$
\begin{gathered}
\int_{0}^{T} \int_{0}^{l}\left[\int_{x}^{l} u_{t} d \xi \int_{x}^{l} v_{t} d \xi-a u v-c \int_{x}^{l} u d \xi \int_{x}^{l} v d \xi+a_{x} u \int_{x}^{l} v d \xi+c_{x} \int_{x}^{l} u d \xi \ell v\right] d x d t \\
=\int_{0}^{T} \int_{0}^{l} f \ell v d x d t-\int_{0}^{l} \int_{x}^{l} \psi d \xi \int_{x}^{l} v(\xi, 0) d \xi d x
\end{gathered}
$$

Заметим, что все слагаемые, входящие в (5), имеют смысл и для функций $u \in \widetilde{\mathscr{H}}_{0}(\mathscr{D})$, $v(x, t) \in \widetilde{\mathscr{H}}_{T}(\mathscr{D})$. Из определения пространства $\widetilde{\mathscr{H}}_{0}(\mathscr{D})$ видно, что если $u \in \widetilde{\mathscr{H}} 0(\mathscr{D})$, то $\int_{x}^{l} u_{t} d \xi \in L_{2}(\mathscr{D})$. Но тогда $\int_{x}^{l} u d \xi \in H^{1}(\mathscr{D})$, а функции из $H^{1}$ имеют след, принадлежащий $L_{2}$. Будем понимать первое из условий Коши в следующем смысле: $\int_{x}^{l} u(\xi, 0) d \xi=$ $\int_{x}^{l} \varphi(\xi) d \xi$.

ОПРЕДЕЛЕНИЕ. Обобщенным решением задачи (1)-(4) будем назьвать функцию $u(x, t) \in \widetilde{\mathscr{H}}_{0}(\mathscr{D})$, удовлетворяющую для всех $v(x, t) \in \widetilde{\mathscr{H}}_{T}(\mathscr{D})$ тождеству (5) и начальному условию

$$
\int_{x}^{l} u(\xi, 0) d \xi=\int_{x}^{l} \varphi(\xi) d \xi
$$




\section{2. Единственность решения.}

Teоpema 1. Eсли $0<a_{0} \leqslant a(x, t) \leqslant A_{0},\left|a_{x}, a_{t}, a_{x x}\right| \leqslant A_{1}, 0 \leqslant c_{0} \leqslant c(x, t) \leqslant C_{0}$, $\left|c_{x}, c_{t}\right| \leqslant C_{1}$, то существует не более одного обобщенного решения задачи (1)-(4).

ДокАЗАТЕльСтво. Пусть $u(x, t) \in \widetilde{\mathscr{H}}_{0}(\mathscr{D})$ - обобщенное решение задачи (1)-(4) с $f(x, t)=0, \varphi(x)=0, \psi(x)=0$. Покажем, что тогда $u(x, t) \equiv 0$ в $\mathscr{D}$.

Возьмем произвольное $\tau \in[0, T]$ и рассмотрим функцию

$$
v(x, t)= \begin{cases}\int_{t}^{\tau} u(x, \eta) d \eta, & 0 \leqslant t \leqslant \tau \\ 0, & \tau \leqslant t \leqslant T .\end{cases}
$$

Определенная таким образом функция принадлежит $\widetilde{\mathscr{H}}_{T}(\mathscr{D})$. Подставим ее в тождество (5), где $f=\varphi=\psi=0$. Так как $v_{t}(x, t)=-u(x, t)$, то

$$
\begin{gathered}
\int_{0}^{T} \int_{0}^{l} \int_{x}^{l} u_{t} d \xi \int_{x}^{l} v_{t} d \xi d x d t=-\int_{0}^{\tau} \int_{0}^{l} \int_{x}^{l} u_{t} d \xi \int_{x}^{l} u d \xi d x d t \\
=-\frac{1}{2} \int_{0}^{l}\left(\int_{x}^{l} u(\xi, \tau) d \xi\right)^{2} d x
\end{gathered}
$$

$\int_{0}^{T} \int_{0}^{l} a u v d x d t=-\int_{0}^{\tau} \int_{0}^{l} a v_{t} v d x d t=\frac{1}{2} \int_{0}^{l} a(x, 0) v^{2}(x, 0) d x+\frac{1}{2} \int_{0}^{\tau} \int_{0}^{l} a_{t} v^{2} d x d t$,

$$
\begin{array}{r}
\int_{0}^{T} \int_{0}^{l} c \int_{x}^{l} u d \xi \int_{x}^{l} v d \xi d x d t=-\int_{0}^{\tau} \int_{0}^{l} c \int_{x}^{l} v_{t} d \xi \int_{x}^{l} v d \xi d x d t \\
=\frac{1}{2} \int_{0}^{l} c\left(\int_{x}^{l} v(\xi, 0) d \xi\right)^{2} d x+\frac{1}{2} \int_{0}^{\tau} \int_{0}^{l} c_{t}\left(\int_{x}^{l} v d \xi\right)^{2} d x d t
\end{array}
$$

$\int_{0}^{T} \int_{0}^{l} a_{x} u \int_{x}^{l} v d \xi d x d t=\int_{0}^{\tau} \int_{0}^{l} a_{x x} \int_{x}^{l} u d \xi \int_{x}^{l} v d \xi d x d t-\int_{0}^{\tau} \int_{0}^{l} a_{x} v \int_{x}^{l} u d \xi d x d t$.

Таким образом, если $u(x, t)$ - решение однородной задачи $(1)-(4)$, то

$$
\begin{gathered}
\int_{0}^{l}\left[\left(\int_{x}^{l} u(\xi, \tau) d \xi\right)^{2}+a(x, 0) v^{2}(x, 0)+c(x, t)\left(\int_{x}^{l} v(\xi, 0) d \xi\right)^{2}\right] d x \\
=\int_{0}^{\tau} \int_{0}^{l}\left(a_{x x} \int_{x}^{l} u d \xi \int_{x}^{l} v d \xi-a_{x} v \int_{x}^{l} u d \xi+c_{x} \int_{x}^{l} u d \xi \int_{x}^{l}(\xi-x) v d \xi\right) d x d t \\
\quad-\frac{1}{2} \int_{0}^{\tau} \int_{0}^{l}\left(a_{t} v^{2}+c_{t}\left(\int_{x}^{l} v d \xi\right)^{2}\right) d x d t
\end{gathered}
$$

Подьнтегральное выражение первого слагаемого в правой части (7) оценим с помощью неравенства Коши и заметим, что

$$
\int_{x}^{l}(\xi-x) v d \xi=\int_{x}^{l} \int_{\xi}^{l} v\left(\xi^{\prime}, t\right) d \xi^{\prime} d \xi
$$


Тогда в силу неравенства Коши-Буняковского

$$
\left(\int_{x}^{l} \int_{\xi}^{l} v d \xi^{\prime} d \xi\right)^{2} \leqslant l \int_{0}^{l}\left(\int_{\xi}^{l} v d \xi^{\prime}\right)^{2} d \xi
$$

и мы получим

$$
\begin{gathered}
\int_{0}^{l}\left[\left(\int_{x}^{l} u(\xi, \tau) d \xi\right)^{2}+a(x, 0) v^{2}(x, 0)+c(x, 0)\left(\int_{x}^{l} v(\xi, 0) d \xi\right)^{2}\right] d x \\
\leqslant \int_{0}^{\tau} \int_{0}^{l}\left[\left(a_{x x}^{2}+\left|c_{t}\right|+l^{2}\right)\left(\int_{x}^{l} v d \xi\right)^{2}\right. \\
\left.\quad+\left(a_{x}^{2}+c_{x}^{2}+\left|a_{t}\right|\right) v^{2}+2\left(\int_{x}^{l} u d \xi\right)^{2}\right] d x d t
\end{gathered}
$$

Введем функцию $w(x, t)=\int_{0}^{t} u(x, \eta) d \eta$. Тогда $v(x, t)=w(x, \tau)-w(x, t), v(x, 0)=w(x$, $\tau), v^{2}(x, t) \leqslant 2 w^{2}(x, \tau)+2 w^{2}(x, t)$ и неравенство (8) примет вид

$$
\begin{aligned}
& \int_{0}^{l}\left[\left(\int_{x}^{l} u(\xi, \tau) d \xi\right)^{2}+a_{0} v^{2}(x, 0)+c_{0}\left(\int_{x}^{l} v(\xi, 0) d \xi\right)^{2}\right] d x d t \\
& \leqslant M \int_{0}^{\tau} \int_{0}^{l}\left[\left(\int_{x}^{l} w(\xi, t) d \xi\right)^{2}+w^{2}(x, t)+\left(\int_{x}^{l} u(\xi, t) d \xi\right)^{2}\right] d x d t \\
& \quad+M \tau \int_{0}^{l}\left[\left(\int_{x}^{l} w(\xi, \tau) d \xi\right)^{2}+w^{2}(x, \tau)\right] d x
\end{aligned}
$$

где $M=2 \max \left\{\left(a_{x x}^{2}+\left|c_{t}\right|+l^{2}\right),\left(a_{x}^{2}+c_{x}^{2}+\left|a_{t}\right|\right), 1\right\}$. Воспользуемся теперь произволом выбора $\tau$. Обозначим $\nu=\min \left\{a_{0}, c_{0}\right\}$. Если $\tau \in[0, \nu /(2 M)]$, то $\nu-M \tau \geqslant \nu / 2$. Обозначим $m=\min \{1, \nu / 2\}$. Тогда

$$
\begin{aligned}
& m \int_{0}^{l}\left[\left(\int_{x}^{l} u(\xi, \tau) d \xi\right)^{2}+w^{2}(x, \tau)+\left(\int_{x}^{l} w(\xi, \tau) d \xi\right)^{2}\right] d x \\
& \quad \leqslant M \int_{\tau} \int_{0}^{l}\left[\left(\int_{x}^{l} u(\xi, t) d \xi\right)^{2}+w^{2}(x, t)+\left(\int_{x}^{l} w(\xi, t) d \xi\right)^{2}\right] d x d t
\end{aligned}
$$

и из леммы 7.1 [8] следует, что

$$
m \int_{0}^{l}\left[\left(\int_{x}^{l} u(\xi, \tau) d \xi\right)^{2}+w^{2}(x, \tau)+\left(\int_{x}^{l} w(\xi, \tau) d \xi\right)^{2}\right] d x=0
$$

для $\tau \in[0, \nu /(2 M)]$. Но тогда для этих значений $\tau \int_{x}^{l} u(\xi, \tau) d \xi=0$ для всех $x \in[0, l]$, поэтому и $u(x, \tau)=0, x \in[0, l], \tau \in[0, \nu /(2 M)]$. Повторяя рассуждения для $t \in[\nu /(2 M)$, $\nu / M]$, убедимся, что $u(x, t)=0$ и на этом промежутке, а через конечное число шагов $u(x, t)=0$ в $\mathscr{D}$.

3. Существование решения. Для доказательства существования решения применим метод Галеркина. Предварительно получим неравенство, которое понадобится для доказательства основной теоремы. 
Лемма. Если $f \in L_{2}(\mathscr{D}), \varphi, \psi \in L_{2}(0, l)$, коэффициенты уравнения (1) удовлетворяют условиям теоремы 1 , то существует постоянная $C>0$ такая, ито для решения $u(x, t) \in \widetilde{C}_{0}^{2}(\mathscr{D})$ справедливо неравенство

$$
\begin{aligned}
& \int_{0}^{l} u^{2}(x, \tau) d x+\int_{0}^{l}\left(\int_{x}^{l} u(\xi, \tau) d \xi\right)^{2} d x+\int_{0}^{l}\left(\int_{x}^{l} u_{t}(\xi, \tau) d \xi\right)^{2} d x \\
& \leqslant \widetilde{C}\left(\|f\|_{L_{2}(D)}^{2}+\|\varphi\|_{L_{2}(0, l)}^{2}+\|\psi\|_{L_{2}(0, l)}^{2}\right)
\end{aligned}
$$

ДокАЗАтЕЛЬСтво. Рассмотрим оператор

$$
\ell\left(u_{t}\right)=\int_{x}^{l}(\xi-x) u_{t}(\xi, t) d \xi .
$$

Преобразуем скалярное произведение $-2\left(\mathscr{L} u, \ell\left(u_{t}\right)\right)_{L_{2}\left(\mathscr{D}_{\tau}\right)}$, где $\mathscr{D}_{\tau}=\{(x, t): 0<x<l$, $0<t<\tau \leqslant T\}$ :

$$
-2\left(\mathscr{L} u, \ell\left(u_{t}\right)\right)_{L_{2}\left(D_{\tau}\right)}=-2 \int_{0}^{\tau} \int_{0}^{l}\left(u_{t t}-\left(a u_{x}\right)_{x}+c u\right) \ell\left(u_{t}\right) d x d t=\sum_{i=1}^{3} I_{i} .
$$

Проинтегрируем $I_{1}$ по частям сначала по $x$, а затем по $t$. Принимая во внимание, что $\int_{0}^{t} u d x=0$ и $u_{t}(x, 0)=\psi(x)$, получим

$$
\begin{aligned}
I_{1} & =2 \int_{0}^{\tau} \int_{0}^{l} u_{t t} \int_{x}^{l}(x-\xi) u_{t} d \xi d x d t \\
& =-\left.2 \int_{0}^{\tau} \int_{x}^{l}(x-\xi) u_{t} d \xi \int_{x}^{l} u_{t t} d \xi\right|_{0} ^{l} d t+2 \int_{0}^{\tau} \int_{0}^{l} \int_{x}^{l} u_{t} d \xi \int_{x}^{l} u_{t t} d \xi d x d t \\
& =\left.2 \int_{0}^{l}\left(\int_{x}^{l} u_{t} d \xi\right)^{2}\right|_{0} ^{\tau} d x-2 \int_{0}^{\tau} \int_{0}^{l} \int_{x}^{l} u_{t} d \xi \int_{x}^{l} u_{t t} d \xi d x d t,
\end{aligned}
$$

и тогда

$$
I_{1}=\int_{0}^{l}\left(\int_{x}^{l} u_{t}(\xi, t) d \xi\right)^{2} d x-\int_{0}^{l}\left(\int_{x}^{l} u_{t}(\xi, 0) d \xi\right)^{2} d x .
$$

Проинтегрируем $I_{2}$ два раза по $x$ :

$$
\begin{aligned}
I_{2} & =2 \int_{0}^{\tau} \int_{0}^{l}\left(a u_{x}\right)_{x} \int_{x}^{l}(\xi-x) u_{t} d \xi d x d t \\
& =\left.2 \int_{0}^{\tau} a u_{x} \int_{x}^{l}(\xi-x) u_{t} d \xi\right|_{0} ^{l} d t+2 \int_{0}^{\tau} \int_{0}^{l} a u_{x} \int_{x}^{l} u_{t} d \xi d x d t \\
& =\left.2 \int_{0}^{\tau} a u \int_{x}^{l} u_{t} d \xi\right|_{0} ^{l} d t-2 \int_{0}^{\tau} \int_{0}^{l} a_{x} u \int_{x}^{l} u_{t} d \xi d x d t+2 \int_{0}^{\tau} \int_{0}^{l} a u u_{t} d x d t .
\end{aligned}
$$

Проинтегрировав последнее слагаемое по $t$, получим

$$
\begin{aligned}
I_{2}= & \int_{0}^{l} a(x, \tau) u^{2}(x, \tau) d x-\int_{0}^{l} a(x, 0) u^{2}(x, 0) d x \\
& -2 \int_{0}^{\tau} \int_{0}^{l} a_{x} u \int_{x}^{l} u_{t} d \xi d x d t-\int_{0}^{\tau} \int_{0}^{l} a_{t} u^{2} d x d t .
\end{aligned}
$$


Таким образом,

$$
\begin{aligned}
-2(\mathscr{L} u, \ell u)_{L_{2}}= & \int_{0}^{l}\left(a(x, \tau) u^{2}(x, \tau)+\left(\int_{x}^{l} u_{t}(\xi, \tau) d \xi\right)^{2}\right) d x \\
& -\int_{0}^{\tau} \int_{0}^{l} a_{t} u^{2} d x d t-2 \int_{0}^{\tau} \int_{0}^{l} a_{x} u \int_{x}^{l} u_{t} d \xi d x d t \\
& -2 \int_{0}^{\tau} \int_{0}^{l} c u \int_{x}^{l}(\xi-x) u_{t} d \xi d x d t \\
& -\int_{0}^{l}\left(a(x, 0) u^{2}(x, 0)+\left(\int_{x}^{l} u_{t}(\xi, 0) d \xi\right)^{2}\right) d x .
\end{aligned}
$$

Учитьвая теперь, что $u(x, t)$ - решение задачи (1)-(4), перепишем последнее равенство так:

$$
\begin{aligned}
& \int_{0}^{l}\left(a(x, \tau) u^{2}(x, \tau)+\left(\int_{x}^{l} u_{t}(\xi, \tau) d \xi\right)^{2}\right) d x \\
& =\int_{0}^{\tau} \int_{0}^{l} a_{t}(x, t) u^{2}(x, t) d x d t+\int_{0}^{l}\left(a(x, 0) \varphi^{2}(x)+\left(\int_{x}^{l} \psi(\xi) d \xi\right)^{2}\right) d x \\
& \quad+2 \int_{0}^{\tau} \int_{0}^{l} a_{x} u \int_{x}^{l} u_{t} d \xi d x d t+2 \int_{0}^{\tau} \int_{0}^{l} c u \int_{x}^{l}(\xi-x) u_{t} d \xi d x d t \\
& \quad-2 \int_{0}^{\tau} \int_{0}^{l} f(x, t) \int_{x}^{l}(\xi-x) u_{t} d \xi d x d t .
\end{aligned}
$$

Применив теперь неравенства Коши к последним трем интегралам правой части этого равенства и учитывая, что

$$
\left(\int_{x}^{l}(\xi-x) u_{t} d \xi\right)^{2}=\left(\int_{x}^{l} \int_{\xi}^{l} u_{t}\left(\xi^{\prime}, t\right) d \xi^{\prime} d \xi\right)^{2} \leqslant \int_{0}^{l}\left(\int_{x}^{l} u d \xi\right)^{2} d x
$$

откуда

$$
\int_{0}^{\tau} \int_{0}^{l}\left(\int_{x}^{l}(\xi-x) u_{t} d \xi\right)^{2} d x d t \leqslant l \int_{0}^{\tau} \int_{0}^{l}\left(\int_{x}^{l} u_{t} d \xi\right)^{2} d x d t
$$

получаем неравенство

$$
\begin{aligned}
m \int_{0}^{l}\left[u^{2}(x, \tau)+\left(\int_{x}^{l} u_{t}(\xi, \tau) d \xi\right)^{2}\right] d x & \\
\leqslant & M \int_{0}^{\tau} \int_{0}^{l}\left[u^{2}+\left(\int_{x}^{l} u_{t}(\xi, t) d \xi\right)^{2}\right] d x d t \\
& \quad+\int_{0}^{\tau} \int_{0}^{l} f^{2} d x d t+l \int_{0}^{l} \psi^{2}(\xi) d x+A_{0} \int_{0}^{l} \varphi^{2}(x) d x
\end{aligned}
$$

где $m=\min \left\{1, a_{0}\right\}, M=\max \left\{2 A_{1}^{2}, 1+2 l\right\}$. Теперь, используя лемму 7.1 ([3]), имеем

$$
\begin{aligned}
& m \int_{0}^{l}\left[u^{2}(x, \tau)+\left(\int_{x}^{l} u_{t}(\xi, \tau) d \xi\right)^{2}\right] d x \\
& \quad \leqslant e^{\frac{M}{m} T}\left[\|f\|_{L_{2}(\mathscr{D})}^{2}+A_{0}\|\varphi\|_{L_{2}(0, l)}^{2}+l\|\psi\|_{L_{2}(0, l)}^{2}\right] .
\end{aligned}
$$

Положив здесь $\widetilde{C}=\frac{M_{1}}{m} \exp (M T / m)$, где $M_{1}=\max \left\{1, A_{0}, l\right\}$, получим неравенство (10). 
TЕОРема 2. Eсли $f(x, t) \in L_{2}(\mathscr{D}), \varphi(x), \psi(x) \in L_{2}(0, l)$ и выполняются условия теоремы 1, то обобщенное решение задачи (1)-(4) существует.

ДокАЗАТЕЛЬСтво. Обозначим $\widetilde{\mathscr{H}}_{0}(0, l)$ замыкание множества

$$
\widetilde{C}_{0}^{2}[0, l]=\left\{w(x) \in C^{2}[0, l], \quad w_{x}(0)=0, \quad \int_{0}^{l} w(x) d x=0\right\}
$$

по норме

$$
\|w\|_{\widetilde{\mathscr{H}}(0, l)}^{2}=\int_{0}^{l}\left(w^{2}+\left(\int_{x}^{l} w d \xi\right)^{2}\right) d x
$$

Так как по определению $\widetilde{\mathscr{H}}_{0}(0, l)$ в нем всюду плотно множество $\widetilde{C}_{0}^{2}[0, l]$, которое является подпространством сепарабельного пространства $C^{2}[0, l]$, то можно выбрать систему линейно независимых функций $\left\{w_{k}(x)\right\}$ из $\widetilde{C}_{0}^{2}[0, l]$, полную в $\widetilde{\mathscr{H}}_{0}(0, l)$.

Обозначим $\widetilde{\mathscr{H}}_{0}^{m}(0, l)$ конечномерное подпространство пространства $\widetilde{\mathscr{H}}_{0}(0, l)$ с бази$\operatorname{com}\left\{w_{k}(x)\right\}_{k=1}^{m}$. Приближенное решение задачи (1)-(4), удовлетворяющее начальным условиям (2) с

$$
\varphi_{m}(x)=\sum_{k=1}^{m} \varphi_{k} w_{k}(x), \quad \psi_{m}(x)=\sum_{k=1}^{m} \psi_{k} w_{k}(x),
$$

будем искать в виде

$$
u_{m}(x, t)=\sum_{k=1}^{m} d_{k}(t) w_{k}(x)
$$

из соотношений

$$
\int_{0}^{l}\left(u_{m t t}-\left(a u_{m x}\right)_{x}+c u_{m}\right) \ell\left(w_{k}\right) d x=\int_{0}^{l} f \ell\left(w_{k}\right) d x
$$

кроме того, $d_{k}(0)=\varphi_{k}, d_{k}^{\prime}(0)=\psi_{k}$, где $\varphi_{k}, \psi_{k}$ - коэффициенты сумм (13), аппроксимирующих при $m \rightarrow \infty$ функции $\varphi(x)$ и $\psi(x)$ соответственно в норме $L_{2}(0, l)$.

Интегрированием по частям преобразуем левую часть (15). Получим

$$
\begin{aligned}
& \sum_{k=1}^{m} d_{k}^{\prime \prime}(t)\left(\int_{x}^{l} w_{k}(\xi) d \xi, \int_{x}^{l} w_{l}(\xi) d \xi\right)_{L_{2}(0, l)} \\
& \quad+\sum_{k=1}^{m} d_{k}(t) \int_{0}^{l}\left(a(x, t) w_{l}(x)-a_{x}(x, t) \int_{x}^{l} w_{l}(\xi) d \xi\right) w_{k}(x) d x \\
& \quad-\sum_{k=1}^{m} d_{k}(t) \int_{0}^{l} c(x, t) w_{k}(x) \int_{x}^{l} \int_{\xi}^{l} w_{l}(\eta) d \eta d \xi d x=f_{k}(t),
\end{aligned}
$$

где $f_{k}(t)=-\int_{0}^{l} f(x, t) w_{k}(x) d x$. Мы получили систему обыкновенных дифференциальных уравнений второго порядка относительно $d_{k}(t)$. Заметим, что из линейной независимости системы функций $w_{k}(x)$ следует линейная независимость функций $\int_{x}^{l} w_{k}(\xi) d \xi$. Действительно, если бы это было не так, то нашлись бы такие $\alpha_{k}$, что

$$
\sum_{k=1}^{m} \alpha_{k} \int_{x}^{l} w_{k}(\xi) d \xi=0, \quad \sum_{k=1}^{m} \alpha_{k}^{2} \neq 0 .
$$


Но для любого $m$

$$
\sum_{k=1}^{m} \alpha_{k} \int_{x}^{l} w_{k}(\xi) d \xi=\int_{x}^{l} \sum_{k=1}^{m} \alpha_{k} w_{k}(\xi) d \xi=0 .
$$

Так как последнее равенство вьполняется для всех $x \in(0, l)$, то

$$
\sum_{k=1}^{m} \alpha_{k} w_{k}(\xi) d \xi=0
$$

что противоречит линейной независимости функций $w_{k}(x)$.

В силу линейной независимости функций $\int_{x}^{l} w_{k}(\xi) d \xi$ определитель матрицы с элементами $\left(\int_{x}^{l} w_{k}(\xi) d \xi, \int_{x}^{l} w_{l}(\xi) d \xi\right)$ отличен от нуля, и система (16) может быть разрешена относительно старших производных и, стало быть, задача Коши для нее с данными $d_{k}(0)=\varphi_{k}, d_{k}^{\prime}(0)=\psi_{k}$ имеет единственное решение. Таким образом, для любого $m$ существует единственная функция $u_{m}(x, t)=\sum_{k=1}^{m} d_{k}(t) w_{k}(x)$. Докажем теперь, что построенная последовательность $\left\{u_{m}(x, t)\right\}$ сходится и ее предел является искомым обобщенным решением задачи (1)-(4). Умножим $(15)$ на $d_{k}^{\prime}(t)$ и просуммируем от 1 до $m$. Получим

$$
\begin{gathered}
\int_{0}^{l}\left(u_{m t t}-\left(a u_{m x}\right)_{x}+c u_{m}\right) \int_{x}^{l}(\xi-x) u_{m t}(\xi, t) d \xi d x \\
=\int_{0}^{l} f(x, t) \int_{x}^{l}(\xi-x) u_{m t}(\xi, t) d \xi d x .
\end{gathered}
$$

Проинтегрируем теперь полученное тождество по $t$ от 0 до $\tau$ и, используя вычисления, проделанные при доказательстве леммы, получим оценку

$$
\begin{aligned}
& \int_{0}^{l} u_{m}^{2}(x, \tau) d x+\int_{0}^{l}\left(\int_{x}^{l} u_{m t}(\xi, \tau) d \xi\right)^{2} d x \\
& \quad \leqslant \widetilde{C}\left(\|f\|_{L_{2}(\mathscr{D})}^{2}+\left\|\varphi_{m}\right\|_{L_{2}(0, l)}^{2}+\left\|\psi_{m}\right\|_{L_{2}(0, l)}^{2}\right) .
\end{aligned}
$$

Так как

$$
\left\|\varphi_{m}(x)\right\|_{L_{2}}^{2}=\sum_{k=1}^{m}\left|\varphi_{k}\right|^{2} \leqslant\|\varphi(x)\|_{L_{2}}^{2}, \quad\left\|\psi_{m}(x)\right\|_{L_{2}}^{2}=\sum_{k=1}^{m}\left|\psi_{k}\right|^{2} \leqslant\|\psi(x)\|_{L_{2}}^{2},
$$

интегрируя теперь обе части этого неравенства по $t$ от 0 до $T$, получим

$$
\left\|u_{m}\right\|_{\widetilde{\mathscr{H}}(\mathscr{D})}^{2} \leqslant C\left(\|f\|_{L_{2}(\mathscr{D})}^{2}+\|\varphi\|_{L_{2}(0, l)}^{2}+\|\psi\|_{L_{2}(0, l)}^{2}\right)
$$

где $C=\widetilde{C} T$. Из (17) следует ограниченность последовательности $u_{m}(x, t)$, и, следовательно, возможность выделить из нее слабо сходящуюся подпоследовательность, за которой мы сохраним прежнее обозначение, к некоторому элементу $u(x, t) \in \widetilde{\mathscr{H}}_{0}(\mathscr{D})$.

Покажем, что $u(x, t)$ и есть искомое обобшенное решение, т.е. для любой функции $v(x, t) \in \widetilde{\mathscr{H}}_{T}(\mathscr{D}) \quad u(x, t)$ удовлетворяет тождеству (5) и начальному условию

$$
\int_{x}^{l} u(x, 0) d x=\int_{x}^{l} \varphi(x) d x .
$$


Действительно, так как

$$
\begin{aligned}
\int_{x}^{l} \varphi_{m} d x-\int_{x}^{l} \varphi d x & =\int_{x}^{l}\left(\varphi_{m}-\varphi\right) d x \leqslant \sqrt{l}\left(\int_{0}^{l}\left(\varphi_{m}-\varphi\right)^{2} d x\right)^{1 / 2} \\
& =\sqrt{l}\left\|\varphi_{m}-\varphi\right\|_{L_{2}} \rightarrow 0, \quad m \rightarrow \infty
\end{aligned}
$$

начальное условие в форме (6) выполняется.

Рассмотрим множество $\mathscr{M}$ всех линейных комбинаций функций $\theta(t) w_{k}(x)$, где

$$
\theta(t) \in C[0, T] \cap C^{2}(0, T), \quad \theta(T)=0 .
$$

Очевидно, что $\theta(t) w_{k}(x) \in \widetilde{C}_{T}^{2}(\mathscr{D})$ для всех $k=1,2, \ldots$ Покажем сначала, что тождество (5) справедливо для любой функции $v(x, t)=\theta(t) w_{k}(x)$. Для этого умножим обе части (15) на $\theta(t)$ и проинтегрируем по $t \in[0, T]$. После несложных преобразований получим

$$
\begin{aligned}
\int_{0}^{T} & \int_{0}^{l}\left(\int_{x}^{l} u_{m t} d \xi \int_{x}^{l} v_{t} d \xi-a u_{m} v-c \int_{x}^{l} u_{m} d \xi \int_{x}^{l} v d \xi\right) d x d t \\
& +\int_{0}^{T} \int_{0}^{l}\left(a_{x} u_{m} \int_{x}^{l} v d \xi+c_{x} \int_{x}^{l} u_{m} d \xi \ell v\right) d x d t \\
= & \int_{0}^{T} \int_{0}^{l} f \ell v d x d t+\left.\int_{0}^{l} \psi_{m} \ell v\right|_{t=0} d x
\end{aligned}
$$

откуда вытекает (5), так как при $m \rightarrow \infty u_{m} \rightarrow u$ слабо в $\widetilde{\mathscr{H}}_{0}(\mathscr{D})$.

Покажем теперь, что $\mathscr{M}$ всюду плотно в $\widetilde{\mathscr{H}}_{T}(\mathscr{D})$. Для этого достаточно установить плотность $\mathscr{M}$ в $\widetilde{C}_{T}^{2}(\mathscr{D})$. Множество $\mathscr{M}$ можно рассматривать как множество всех линейных комбинаций функций $\widetilde{w}_{k}(x) \theta(t)$, где $\theta(t) \in C^{1}[0, T], \theta(T)=0, \widetilde{w}_{k}(x)$ - ортонормированный базис в $\mathscr{H}_{0}(0, l)$ в скалярном произведении $(f, g)=\int_{0}^{l}\left(f g+\int_{x}^{l} f d \xi \int_{x}^{l} g d \xi\right) d x$, полученньй в результате ортонормирования системы функций $w_{k}(x)$.

Пусть $\eta(x, t)$ - произвольная функция из $\widetilde{C}_{T}^{2}(\mathscr{D})$. Так как для любого $t \in[0, T] \eta(x, t)$, $\eta_{t}(x, t) \in L_{2}(0, l)$, их можно разложить в метрике $L_{2}(0, l)$ в сходящиеся ряды

$$
\eta(x, t)=\sum_{k=1}^{\infty} \eta_{k}(t) \widetilde{w}_{k}(x), \quad \eta_{t}(x, t)=\sum_{k=1}^{\infty} \eta_{k}^{\prime}(t) \widetilde{w}_{k}(x)
$$

где

$$
\eta_{k}(t)=\int_{0}^{l} \eta(x, t) \widetilde{w}_{k}(x), \quad \eta_{k}^{\prime}(t)=\int_{0}^{l} \eta_{t}(x, t) \widetilde{w}_{k}(x)
$$

при этом

$$
\sum_{k=1}^{\infty}\left(\eta_{k}^{2}(t)+\eta_{k}^{\prime 2}(t)\right)=\int_{0}^{l}\left(\eta^{2}(x, t)+\eta_{t}^{2}(x, t)\right) d x
$$


Обозначим через $\eta_{N}(x, t)=\sum_{k=1}^{N} \eta_{k}(t) \widetilde{w}_{k}(x)$ частичную сумму ряда Фурье. Рассмотрим

$$
\begin{aligned}
\| \eta- & \eta_{N}\left\|_{\widetilde{\mathscr{H}}(0, l)}^{2}+\right\| \int_{x}^{l}\left(\eta_{t}-\eta_{N t}\right) d \xi \|_{L_{2}(0, l)}^{2} \\
& =\int_{0}^{l}\left[\left(\eta-\eta_{N}\right)^{2}+\left(\int_{x}^{l}\left(\eta-\eta_{N}\right) d \xi\right)^{2}\right] d x+\int_{0}^{l}\left(\int_{x}^{l}\left(\eta_{t}-\eta_{N t}\right) d \xi\right)^{2} d x \\
& \leqslant(1+l)\left(\left\|\eta-\eta_{N}\right\|_{L_{2}(0, l)}^{2}+\left\|\eta_{t}-\eta_{N}\right\|_{L_{2}(0, l)}^{2}\right)=(1+l) \sum_{k=1+N}^{\infty}\left(\eta_{k}^{2}(t)+\eta_{k}^{\prime 2}(t)\right) .
\end{aligned}
$$

В силу (18)

$$
\sum_{k=1+N}^{\infty}\left(\eta_{k}^{2}(t)+\eta_{k}^{\prime 2}(t)\right) \rightarrow 0, \quad N \rightarrow \infty
$$

Поэтому

$\left\|\eta-\eta_{N}\right\|_{\widetilde{\mathscr{H}}(\mathscr{D})}^{2}=\int_{0}^{T}\left(\left\|\eta-\eta_{N}\right\|_{L_{2}(0, l)}^{2}+\left\|\int_{x}^{l}\left(\eta_{t}-\eta_{N t}\right) d \xi\right\|_{L_{2}(0, l)}^{2}\right) d t \rightarrow 0, \quad N \rightarrow \infty$.

Утверждение доказано.

ЗАмЕчАниЕ. Полученная в лемме априорная оценка справедлива и для решения смешанной задачи для уравнения (1) с граничным условием Дирихле $u(0, t)=0$ и нелокальным интегральным условием с весом

$$
\int_{0}^{l} x u(x, t) d x=0
$$

\section{СПИСОК ЦИТИРОВАННОЙ ЛИТЕРАТУРЫ}

[1] Самарский А. А. О некоторых проблемах теории дифференциальных уравнений // Дифференц. уравнения. 1980. Т. 16. №11. С. 1925-1935.

[2] Cannon J. R. The solution of the heat equation subject to the specification of energy // Quart. Appl. Math. 1963. V. 21. P. 155-160.

[3] Ионкин Н. И. Решение одной краевой задачи теории теплопроводности с неклассическим краевым условием // Дифференц. уравнения. 1977. Т. 13. № 2. С. 294-304.

[4] Нахушев А. М. Об одном приближенном методе решения краевых задач для дифференциальных уравнений и его приложения к динамике почвенной влаги и грунтовых вод // Дифференц. уравнения. 1982. Т. 18. №1. С. 72-81.

[5] Гордезиани Д. Г., Авалишвили Г. А. Решения нелокальных задач для одномерных колебаний среды // Матем. моделир. 2000. Т. 12. № 1. С. 94-103.

[6] Bouziani A. Solution forte d'un problème mixte avec conditions non locales pour une classe d'equations hyperboliques // Bull. CI. Sci. Acad. Roy. Belg. 1997. V. 8. P. 53-70.

[7] Beilin S. Existence of solutions for one-dimensional wave equations with nonlocal conditions // Electronic J. of Diff. Equations. 2001. V. 76. P. 1-8.

[8] Гординг Л. Задача Коши для гиперболических уравнений. М.: ИЛ, 1961.

Самарский государственный университет 